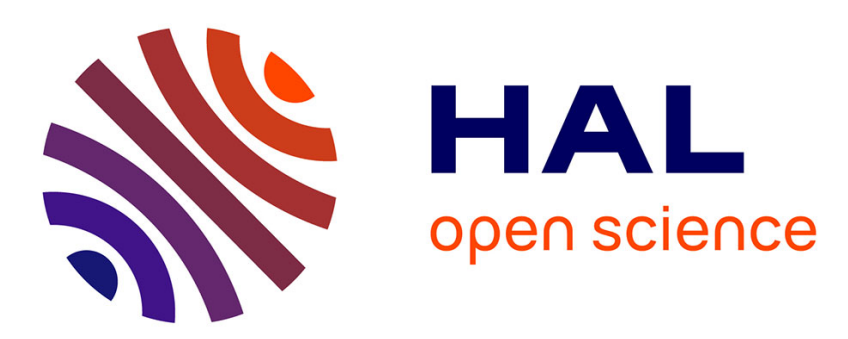

\title{
Degradation state model-based prognosis for proactively maintaining product performance
}

\author{
Benoît Iung, Maxime Monnin, Alexandre Voisin, Pierre Cocheteux, Eric \\ Levrat
}

\section{- To cite this version:}

Benoît Iung, Maxime Monnin, Alexandre Voisin, Pierre Cocheteux, Eric Levrat. Degradation state model-based prognosis for proactively maintaining product performance. CIRP Annals - Manufacturing Technology, 2008, 57 (1), pp.49-52. 10.1016/j.cirp.2008.03.026 . hal-00463121

\author{
HAL Id: hal-00463121 \\ https://hal.science/hal-00463121
}

Submitted on 11 Mar 2010

HAL is a multi-disciplinary open access archive for the deposit and dissemination of scientific research documents, whether they are published or not. The documents may come from teaching and research institutions in France or abroad, or from public or private research centers.
L'archive ouverte pluridisciplinaire HAL, est destinée au dépôt et à la diffusion de documents scientifiques de niveau recherche, publiés ou non, émanant des établissements d'enseignement et de recherche français ou étrangers, des laboratoires publics ou privés. 


\title{
Degradation state model-based prognosis for proactively maintaining product performance
}

\author{
B. lung (2), M. Monnin, A. Voisin, P. Cocheteux, E. Levrat \\ CRAN, Nancy-Université, CNRS, Boulevard des Aiguillettes B.P. 239 \\ F-54506 Vandœuvre lès Nancy
}

\begin{abstract}
Maintenance plays now a critical role in manufacturing for achieving important cost savings and competitive advantage while preserving product conditions. It suggests moving from conventional maintenance practices to predictive strategy. Indeed the maintenance action has to be done at the right time according the component Remaining Useful Life (RUL) assessed by a prognosis process. The accuracy of the RUL is mainly depending on the relevance of the component degradation model used for prediction. In that way, this paper aims at discussing an efficient degradation model taking into account the operational conditions, the health monitoring and the maintenance actions. This model is based on discrete states associated with the degradation levels, and on a cumulative function modelling the transition time between successive states. The model is implemented by means of Stochastic Activity Networks (SAN). The feasibility and added value of such degradation models for prognosis is then highlighted through experimentations made on manufacturing TELMA platform.
\end{abstract}

\section{Keywords}

Maintenance, Failure Model, Prognosis

\section{INTRODUCTION}

Today, Maintenance is one of the major services associated with product life cycle management [1]. Indeed efficient maintenance can mainly contribute to control the conditions of the products for providing the functionality required by customers while ensuring sustainability and maintaining appropriate corporate profit. The challenge is thus to decide maintenance actions on components in the way to continuously reduce and eliminate costly unscheduled downtime and unexpected breakdowns while mastering the product deteriorations (capitalintensive operation). It suggests to change the focus of maintenance policies traditionally based on "fail and fix" practices towards "condition-based vs. predictive" strategies performed only when a certain level of component degradation (impacting profit/product conditions) occurs. The anticipation action which characterises this new strategy is mainly based on a prognosis module processing monitored and/or historical data. In consistent with ISO13381, the prognosis aims at foreseeing how a component will evolve from its current degraded state until its failure and then until the system's breakdown (performance level): to predict its Remaining Useful Life (RUL) [2]. Therefore the accuracy of the RUL plays an essential role in the full realization of the potentials of predictive maintenance [3]. In that way, one major influence factor is represented by the degradation process (model) of the component in terms of initiation (current state) but also progression (by integrating the future such as wear, corrosion...). When the degradation process cannot be known and then formalised by analytical expression (i.e. too complex; higher abstraction level), it is most of the time modelled with several discrete states (i.e. Markov stochastic process) representative of different levels of degradation (acceptable levels; over threshold). Nevertheless, most of these models take into account only simplified parameters within the expression conditioning the evolutions between states. To face this issue, the degradation model discussed in this paper and consistent with the prognosis methodology developed in [2], takes into account, in a unified way, the operational conditions, the health monitoring and the maintenance actions. The development of the degradation model follows a generic approach which can be applied as well as for the degradation of a component as for a function, a sub-system or a system. This model is based both on discrete states associated with the degradation levels, and on a cumulative function modelling the transition time between successive states. The main originality of this work is to structure the degradation discrete states to specific levels defined in accordance with (a) maintenance purpose, (b) particular performances on the components or on each manufacturing system layer, or (c) product conditions. Health monitoring allows adjusting the model according to the current state of the product and/or the manufacturing system. The cumulative function is used to model the impacts of operational and environmental conditions but also maintenance actions on the time between degradation states.

All this original work is firstly justified from a brief statement on degradation modelling made on section 2 and secondly detailed on section 3 . Section 4 underlines the implementation of the model by means of Stochastic Activity Networks (SAN) and its use for the prognosis of a specific component of our TELMA platform. Then section 5 discuss the results of the experimentation and finally conclusion and prospective are developed in section 6 .

\section{PROBLEM STATEMENT ON DEGRADATION MODEL FOR PROGNOSIS}

The degradation modelling is structured mainly on two types of model: continuous and discrete. In the first case, degradations are represented most of the time by stochastic processes (e.g. Wiener's process [4]). The degradation can also be materialised as an accumulative phenomenon (e.g. wear) where it is the degradation increasing which follows a stochastic process, e.g. a Gamma law in [5]. [6] use a vector, representing covariables to take into account in the authors' degradation model of the variations of the operational conditions. 
In the discrete case, the degradation levels are represented with different states by using formalisms such as Markov chains. These states are generally defined by a physical reality whereas the transition from a state to another one is supported by a stochastic way (i.e. failure rate). [7] exploits this principle to develop a state for each output flow of a water pipe.

In summary, compared to the RUL calculation at a functional or system level as it is required to control the product conditions, a continuous process-based degradation model is almost impossible to (analytically) obtain due to the abstraction and/or the complexity levels. In consistence with the standard ISO 13381-1, a discrete model is more adapted for solving this complexity but only while taking into account in the model, the influential factors of degradation such as the impact of maintenance actions or the variation of the operational conditions. This way constitutes, compared to existing work, the originality of our approach which is detailed and illustrated in the following sections.

\section{DEGRADATION \\ STATE MODEL-BASED} PROGNOSIS

As the degradation model objective is to support efficient prognosis, the following hypothesis can be formulated:

- Degradation process before failure is considered,

- According to ISO 13381-1, prognosis must integrate future operating condition and maintenance actions,

- $\quad$ Since system prognosis is required, a hierarchical approach is applied to combine systems, subsystems, component aspects and multiple degradation processes ones,

- Performance levels are defined to translate the objective of each decomposition level and linked to degradation process,

- The results of the prognosis have to be used by a decision-making module [8] (predictive Maintenance) for which the decision is mainly based on different states of the component/system known in advance. A discrete state model is well adapted to support this issue.

From these assumptions, our methodological objective is to propose an engineering for the modelling of a system degradation which is integrating different points of view such as the performance (functional aspect), the degradation modes (organic aspect) and the operation on the whole of the critical components of a system. The first step of this engineering consists in defining a component model which is implementing all these points of view. Then this model can be used at higher abstraction levels for developing sub-system/system degradation models. Whatever the level, the modelling principles are similar. In that way, the paper is focusing on component modelling with the following modelling assumptions:

- A component can be identified by a (global) performance which is representative with its goal. The component degradation leads to a performance decreasing.

- The maintenance actions, done on the component, are defined from its state (i.e. current degradation modes and their evolutions).

For formalising all these points of views, the component model is developing both from hierarchic modular discrete state models (HMDSM) and continuous models. The different models are represented in figure 1 (with their relationships) and described as follows:

- State model of the component performance (HMDSM),
- State model of the degradation modes (HMDSM),

- Model of the impact of the operational/environmental conditions on the dynamics of the degradation mode (continuous model)

- Model of the impact of the maintenance actions on the evolution/state of the degradation mode

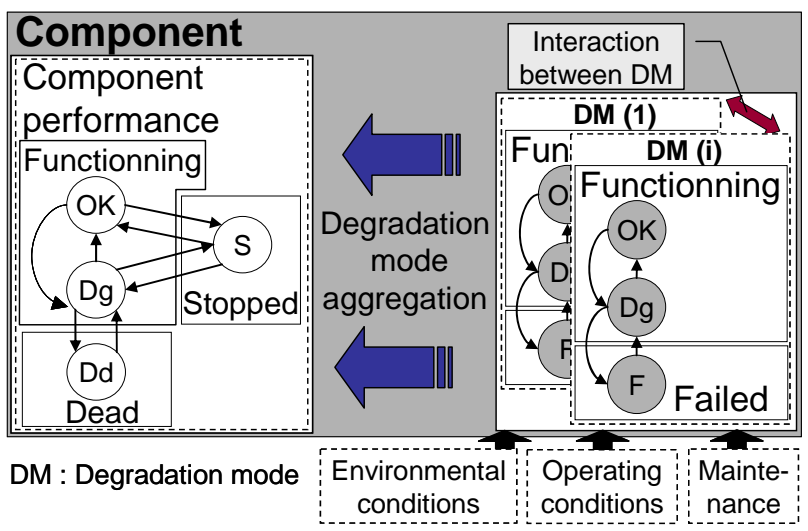

Figure 1 : Component models

\subsection{State model of the component performance}

This model aims at representing, for the operation phase, all the possible states of the component performance (and not the component state). The state and the performance level are called by the same terms and they can be described with 4 states:

- OK: nominal - The component function is fulfilled with a sufficient level of performance leading to consider the component in its nominal state.

- Dg: Degraded - The component function is not satisfied now with a sufficient performance level, but the component is still functioning (in a degraded state). Several degradation modes can be the causes of this state.

- Dd: Dead - The component function is not satisfied now due to a performance level too low. The component is considering in non functioning mode (failed state).

- S: Stopped - The component function cannot be fulfilled because the component functioning is voluntarily stopped. In this state, a level of performance cannot be allocated (no meaning).

The transitions between the $\mathbf{O K}$, Dg and Dd states are fired according the states defined on the degradation mode(s). Thus it is possible to have in the same time several degradation modes in progress and to define their interactions for generating the component state (acceleration, compensation...). The transitions between $\mathrm{OK} / \mathrm{Dg} / \mathrm{Dd}$ and $\mathbf{S}$ states are managed by the operation planning expected in the future of the component (production, maintenance, stop...).

\subsection{Model on the evolution state of the degradation modes}

The performance level of the component is evolving in the time according to one or several degradation processes which are related to the component use. For a component such as a V-Belt, many degradation modes leading to a degraded state exists (thickness, elongating, surface quality...). A degradation mode is supposed to be observable by mean of an indicator. A degradation level is in phase with a performance state of a component. Nevertheless, these states cannot be directly mapped from the states defined in §3.1. A refinement is needed and must follow certain rules. 
At least, three states have to be identified for the degradation mode (as for the component) allowing to be connected to the OK, Dg and Dd states of the component (figure 2). The 3 states of the component can be considered as state classes for the degradation mode. These classes being defined in accordance to component function (performance for supporting the finality), it is logical that the states of the degradation modes have to be referred to them.

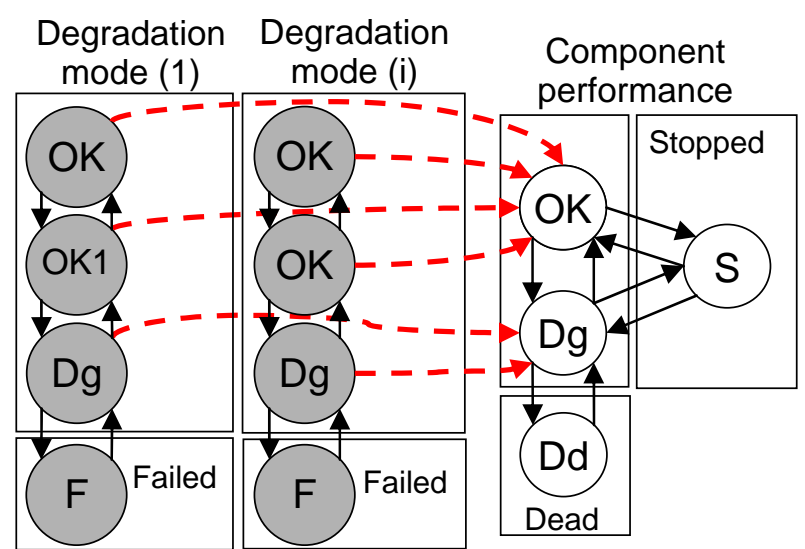

Figure 2: Mapping between component states and degradation states

For the class "OK" of the component, it is proposed to add 2 states for the degradation mode (OK and OK1). These 2 modes are corresponding on one hand, to the quite optimal functioning where the degradation does not appear and on the other hand, to a functioning which starts to be degraded but for which the performance is sufficient and a maintenance action will be necessary in the future to maintain the finality. In this case, the creation of an additional state is directly linked to the maintenance need.

For the class "Degraded", several considerations can help the development of additional states. First, the degradation impact on the component, i.e. even if the performance is degraded, a maintenance action (other than a change) can restore the component in a nominal state whereas a certain threshold is over, it is not true. Second, the most adapted maintenance action, for a specific case, is not the same one.

Finally, for all the classes, if an interaction is taking into account between degradation modes, it can be necessary to create additional classes which will allow, for example, to trigger another degradation mode when a mode reaches a certain state.

These states are defined in relation to the degradation indicator. Thus, several thresholds have to be defined according to the states of the component performance. These thresholds could be issued from an analysis of degradation mode enabling to identify the impact of the degradation mode on the component performance. For example, in the case of the V-Belt elongating, it is necessary to identify four thresholds ( $T h, T h_{c}, T h_{1}, T h_{2}$ ) on the length $L$ of the belt with:

- $\mathrm{L}<$ Th means that the drive is nominal

- $\mathrm{L}>$ Th means that the drive is degraded

- $\mathrm{L}>\mathrm{Th}_{\mathrm{c}}$ means that the drive is lost.

- $\mathrm{Th}_{1}<\mathrm{L}<\mathrm{Th}$ : A maintenance action is needed to preserve the performance,

- $T h<T h_{2}<L<T h_{c}$ : The degradation of the belt surface state is due to an important slip.

An accumulation function is used to manage the transitions between these states. The increment of this function (at each step of time) takes into account the ageing and the impact of the operational and environmental conditions:

$$
\begin{aligned}
& d S_{t}=f\left(d S_{t}^{O p}, d S_{t}^{E n}, t\right) \\
& S_{t}=S_{t-1}+d S_{t}
\end{aligned}
$$

The accumulation function underlines the fact that degradation results from the use of the resource, and that the degradation dynamics is conditioned by the expected production scenarios. The transitions are fired when the variable of accumulation exceeds the thresholds defines previously between the states.

\subsection{Impact of the operational/environmental conditions on the dynamics of the degradation}

The objective of the models (one per type of conditions) built on the same principles is to formalise the contribution of the operational and environmental conditions to the degradation dynamics. The interest of this approach is to enable evolutions according to the component wear and also in the form of shocks such as those developed during startings, shifts of production, etc. The general form of the increment between two states given for a degradation mode is presented in table 1 knowing that these two forms can be:

\begin{tabular}{|l|l|}
\hline $\begin{array}{l}\text { Standard } \\
\text { increment }\end{array}$ & $d S_{t}^{i}=g_{u}\left(C_{1}^{i}, \ldots, C_{n_{i}}^{i}\right)$ \\
\hline $\begin{array}{l}\text { Operating or } \\
\text { environne- } \\
\text { mental shock : }\end{array}$ & $\left\{\begin{array}{l}d S_{t}^{i}=g_{c}\left(C_{1}^{i}, \ldots, C_{n_{i}}^{i}\right) \text { if } \delta\left(C_{1}^{i}, \ldots, C_{n_{i}}^{i}\right)=1 \\
d S_{t}^{i}=0 \quad \text { if } \delta\left(C_{1}^{i}, \ldots, C_{n_{i}}^{i}\right)=0\end{array}\right.$ \\
\hline
\end{tabular}

Tableau 1: Different forms of the increment.

With $i=\{O p, E n\}$ taken into account the evolution model based on operational or environmental conditions, $C_{j}^{i}$ the $j^{\text {th }}$ variables describing the operational/environmental conditions, $n_{i}$ the number of these variables, $d S_{t}^{i}$ the increment contributing to the degradation mode of degradation, $g_{u}$ and $g_{c}$ the functions defining how the variables contribute to the increment according to a wear function or shock function depending then on a change of conditions and $\delta$ the function defining when a shock occurs.

\subsection{Model of the impact of the maintenance actions on the evolution state of the degradation mode}

This model allows reflecting the impact of the maintenance actions on the degradation modes. It integrates the impacts of maintenance actions which will be different according to the technology of the component considered. The goal of this model consists in modifying the evolution conditions of models dedicated to degradation mode. The potential modifications consecutive to a maintenance action are: a change of state which materialises, for example, a change (a replacement); or a change of parameters governing the dynamics of the state changes which materialises, for example, a greasing of a bearing.

\section{APPLICATION TO PROGNOSIS ON TELMA PLATFORM}

The feasibility and interest of the algorithm are considered in relation to an application case extracted from TELMA platform already explained in [9]. TELMA allows to unwind metal bobbins and one subset of TELMA is dedicated to the strip accumulation. In this subset a V-belt connects the electrical engine to the system which pulls the strip.

The degradation process under consideration is the elongating of the $\mathrm{V}$-belt. The indicator of the process is the length of the belt. The main influence variable 
considered is the rotation speed which translates into product parameter as the production speed.

For the DM model, 5 states are defined for the 3 classes:

- OK class: OK et OK1,

- Dg class: Dg1, which corresponds to a loss of the function, and Dg2, which corresponds to a loss of the function plus an irreversible degradation,

\section{- $\quad$ Dd class: $\mathbf{F}$}

The thresholds between states can be identified with trials or constructor's recommendation.

A unique operational condition model is required defining the effect of the production speed $(V)$ on the degradation. The increment is defined as:

$$
d S_{t}^{O p}=g_{w}(V)=a_{k, l} V_{t}^{2}+b_{k, l} V_{t}+c_{k, l}
$$

Polynomial parameters $\left(a_{k, l}, \quad b_{k, l}\right.$ and $\left.c_{k, l}\right)$ depend on transition between state $k$ and state $l$.

The maintenance model includes one maintenance action: the belt replacement. This action makes the maintenance model changing the current state of the degradation model to the OK state.

The models (component, degradation, maintenance and operating condition) are implemented by means of SAN. SAN models are a stochastic generalization of Petri Nets and are more flexible than most other Petri Nets extensions. Structurally, SAN are composed of activities, places, input and output gates. Input and output gates allow complex dependent behavior to be modeled. The models were developed with software tool Möbius [10].

\section{RESULTS - DISCUSSION}

The prognosis aim is characterized by mean of two Remaining Useful Lives (RUL): the component RUL $\left(R U L_{c}\right)$ and the function RUL $\left(R U L_{f}\right)$. RUL $L_{c}$ corresponds to the duration after which the component is out of order (i.e. component model Dd state). $\mathrm{RUL}_{\mathrm{f}}$ corresponds to the duration after which the finality of the function no longer holds (i.e. component model Dg state). To highlight the effectiveness of the approach, 6 simulation scenarios have been defined according to 3 production speeds $\left(V_{1}<\right.$ $V_{2}<V_{3}$ ) and one possible maintenance action with a 2500-hour simulation horizon: (1) constant production speed $\mathrm{V}_{1}$ and no maintenance, (2) constant production speed $V_{2}$ and no maintenance, (3) constant production speed $V_{3}$ and no maintenance, (4) $V_{3}$ for $450 \mathrm{~h}$, then $V_{2}$ for $1000 \mathrm{~h}$, and $V_{1}$ for $550 \mathrm{~h}$, with no maintenance, (5) the same operating condition with a maintenance action at $t=450 \mathrm{~h}, \quad(6)$ the same operating condition with a maintenance action at $t=688 \mathrm{~h}\left(\mathrm{RUL}_{\mathrm{f}}\right.$ obtained for scenario \#4). The results are reported in figure 3.

Scenarios 1 to 3 highlight the impact of operating condition on $\mathrm{RUL}_{\mathrm{c}}$ and $\mathrm{RUL}_{\mathrm{f}}$ : an increasing production speed decrease the RULs. Scenario 4 has been used to demonstrate the ability of the model to tackle specific changing operating condition. Scenario 5 shows the effect of the maintenance action by improving both $\mathrm{RUL}_{c}$ and $\mathrm{RUL}_{f}$ with regard to scenario 4. Finally, scenario 6 highlights the impact of maintenance action date on the RULs. This simple example has demonstrated the ability of the model to integrate operating condition and maintenance action in the RULs assessment.

\section{CONCLUSION AND OUTLOOK}

In this paper, a generic approach for developing a degradation model is proposed in order to improve the prognosis efficiency. The degradation model proposed relies on a unified modelling approach to account for operating conditions, environment conditions and maintenance actions. An application on a specific process ( $V$-belt) of TELMA platform has been carried out to show the feasibility of this approach. Based on these results, further developments should be implemented to demonstrate the real efficiency and added value of this approach. It should consist mainly in improving the accuracy of the degradation model by integrating complex stochastic degradation processes and enhancing the maintenance action modelling, considering multiple degradation modes from a component point of view, developing the approach to a global system (multicomponents) at different abstraction levels.

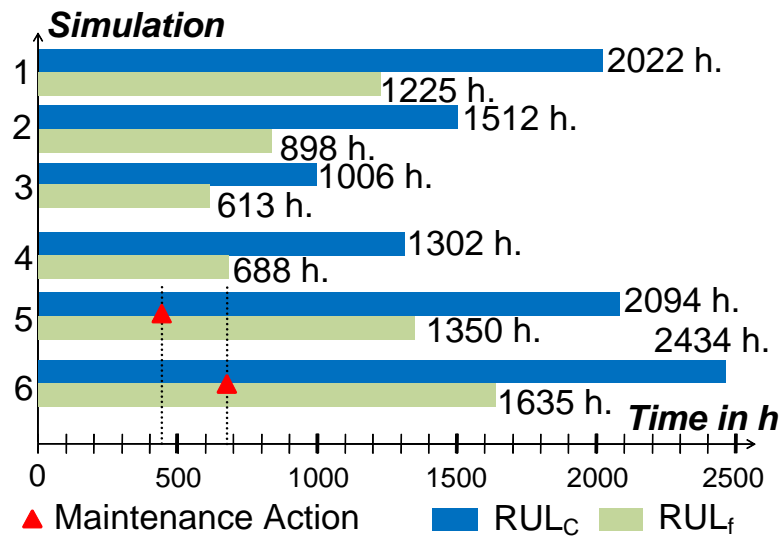

Figure 3: Simulation results

\section{REFERENCES}

[1] Takata, S., Kimura, F., Van Houten, F.J.A.M., Westkämper, E., Shpitalni, M., Ceglarek, D., Lee, J., 2004, Maintenance: Changing Role in Life Cycle Management, Annals of the CIRP, 53/2, 643-656.

[2] lung, B., Veron, M., Suhner, M.C., Muller, A., 2005, Integration of maintenance strategies into prognosis process to decision-making aid on system operation, Annals of the CIRP, 54/1, 5-8.

[3] Liu, J., Djurdjanovic, D., Ni, J., Casoetto, N., Lee, J., 2007, Similarity based method for manufacturing process performance prediction and diagnosis, Cil, 58, 558-566.

[4] Barker, C.T., Newby, M.J., 2008. Optimal nonperiodic inspection for a multivariate degradation model, Reliability Engineering \& System Safety (RESS), doi:10.1016/j.ress.2007.03.015.

[5] Saassouh, B., Dieulle, L., Grall, A., 2007, Online maintenance policy for a deteriorating system with random change of mode, RESS, 92, 1677-1685.

[6] Jiang, R., Jardines, A.K.S., 2008, Health state evaluation of an item: A general framework and graphical representation, RESS, 93, 89-99.

[7] Tan, C.M., Raghavan, N., 2008, A framework to practical predictive maintenance modeling for multistate systems, RESS, doi:10.1016/j.ress.2007.09. 003.

[8] lung, B., Levrat, E., Thomas, E., 2007, 'Odds Algorithm'-based Opportunistic Maintenance Task Execution for Preserving Product Conditions, Annals of the CIRP, 56/1, 13-16.

[9] Levrat, E., lung, B., 2007, TELMA: A full emaintenance platform, Proceedings of WCEAM/CM 2007, Harrogate, June 11-14.

[10] Beaudet, S. T., Courtney, T., Sanders, W. H., 2006, A Behavior-Based Process for Evaluating Availability Achievement Risk Using Stochastic Activity Networks, Proceedings of RAMS 2006, Newport Beach, California, January 23-26. 